\title{
EFFICIENT MODELLING OF HEIGHT DATUM BASED ON GIS
}

\author{
Qinghua Qiao \\ Key Laboratory of Geo-Informatics of State Bureau of Surveying and Mapping \\ Chinese Academy of Surveying and Mapping, Beijing, China - \\ qiaoqh@casm.ac.cn
}

KEY WORDS: Height Datum, GIS, interactive visualization

\begin{abstract}
:
Since the proposal of Digital Earth, its research and applications are continuing to be deepened, and now Smart City is more indepth implementation of the Digital Earth. The unification of global or regional vertical datums has always been one of the main geodesy studies to achieve Smart City, as Smart City must first realize the seamless integration of multi-source geo-dataset. This paper introduces spatio-temporal data management and interactive visualization into the entire process of vertical datum modelling. We demonstrate that the efficiency of modelling is greatly improved. In this paper GIS database is used to manage all dataset such as tidal station data, gravity field model data, GPS leveling data, and vertical datum data for evaluation and analysis. We use geographic information visualization technique to graphically display the results, together with the interactive browsing, to convert tedious digital information to easy-to-understand images. Consequently, researchers can quickly and comprehensively grasp the macro and micro information. Finally, an efficient and interactive prototype operating platforms for vertical datum modeling is constructed based on GIS.
\end{abstract}

\section{INTRODUCTION}

Since Gore brought the concept of Digital Earth, spatial data applications are growing faster than before in popularity. Meanwhile, with social progress and technological development, there is a growing emphasis on GIS industry, more and more deeply combining applications continue to emerge. These applications require high precision and current spatial data. But on the one hand, there is a serious shortage in government funding in space data acquiring and updating. On the other hand, there is no uniform standard for data producing using multisector or multi-unit. For example, the State Bureau of Surveying and Mapping is responsible for basic geographic information update, while other geographic information are produced by other departments, companies and social organizations themselves. When the industries began to adopt GIS in their business, we found that valuable data is not enough. This requires a multi-sector data fusion, which can better serve the industry application. In recent years, digital city is gradually upgrading to intelligent city. The application problem of multisource spatial data fusion is increasingly urgent, so the relative scarcity of geographic data is one of the biggest limitations of the rapid development of the geographic information industry. To solve this problem, we firstly have to establish an unified vertical datum. This article will introduce temporal GIS technology into the entire process of vertical datum conversion, and to accelerate the process of vertical datum modelling.

\subsection{Temporal GIS}

Temporal GIS is a comprehensive application technology based on temporal database, GIS, and artificial intelligence, and is used to study the nature laws of birth, growth, survival until death for objects in space and time world (Wang jiayao, 2004). Generally, temporal GIS have basic process functions, such as spatio-temporal data acquire, store, manage, analyze and display. According to statistics, in the information which human contact with, more than $80 \%$ are associated with spatial position.
Therefore, GIS has a wide range of applications in our daily work and lives. When the target problem is complex, such as larger amount of data and data types, higher efficiency requirements, GIS can play a important role in this case. Geographic information systems made an astonishing development in the last 30 years, which is widely used in resource surveys, environmental assessments, disaster prediction, land management, urban planning, telecommunications, transportation, military, public security, water and power, public facilities management, agriculture, forestry animal husbandry, statistics, business finance almost all areas of whole society. Similarly, the studies of vertical datum modelling related to efficient integration and management for multi-source, multi-temporal state, multi-semantic, and multiscale of spatial information, interactive dynamic adjustment and efficient result data visualization. With temporal GIS, these problems can be better solved.

\subsection{Vertical datum transformation model}

On the one hand, the construction of our spatial data infrastructure spans a longer time, at different times using different techniques and standards. On the other hand, the data in same period is often not full covered, typically for large-scale topographic maps. Early, we conducted national surveying and mapping by using traditional leveling. With the development of technology and equipment, surveying and mapping gradually shift to digital elevation benchmarks based on GNSS (Li jiancheng, 2012). In the process of integration using of multiple-period data, the problem we must firstly solve is the vertical datum unification. The spatial data production which is not led by professional mapping departments is more serious, and is more difficult in the unification. Vertical Datum modelling plays an important role in terms of multi-source spatial data fusion.

Because China uses normal height system, so we must first convert the original vertical datum to normal height systems, usually it can be achieved by solving the height anomaly. 
Secondly, by using fitting method vertical datum conversion can be realized. Now, several studies are conducted using analytical, moving surfaces, multi-faceted function, etc, (Yang yuanxi, 2001; Yang qingzhen, 2012; Zhang xiaohong, 2007).

\section{MODELLING METHODS}

\subsection{Height anomaly solving}

Currently, the method for solving the height anomaly is divided into three categories: gravity field model, geometric standard fitting and combination method. The first method uses gravity field model, such as EGM96, to compute height anomaly based on point information directly. This method is reliable and practical, but requires sufficient gravity data with high precision, and the precision of the result is usually not high enough. Geometric standard fitting method uses known and limited elevation outliers to measure any point throughout the target area, commonly used methods include the least squares method, multi-faceted functions, kriging, mathematical surface method, etc. But if only using fitting method, the accuracy of the results is often poor, especially in complex terrain areas. In the actual mapping, a combination of both methods is usually used (Guo chunxi, 2013).

\subsection{Height Fitting}

In the vertical datum conversion process, researchers often use methods such as kriging interpolation, polynomial curve fitting, inverse distance weighting, multifaceted function to achieve height fitting. Kriging algorithm considers the spatial distribution of elevation variation, for a location to be measured, as long as its influential area is determined, we can use the sample height in this region to estimate the height of point with no measurement A large number of applications show that the interpolation result of this approach has higher reliability. Inverse distance weighted method is simple, but it is difficult to determine its influence scope which has effect on target location. Polynomial curve fitting is an interpolation method commonly used, but there are some human factors exist on how to select nodes and polynomial time. Principle of multifaceted function is that arbitrary sleek mathematical surface can be approached by a series of regular math surface with arbitrary precision. Since natural terrain is very complex, a single interpolation method usually cannot meet the accuracy requirements in practice. Currently, actual data production and scientific research usually use a combination of the above methods. But how to carry out new combination and quickly adjust the weight parameters, is greatly impacting on overall efficiency of vertical datum conversion studies.

\section{MULTISOURCE DATA PRE-PROCESS AND MANAGEMENT}

\subsection{Data pre-process}

Vertical Datum Conversion modelling need to use a variety of data sources, such as spatial data infrastructure, tidal station data, gravity field model data, GPS leveling data, height datum data, etc. Usually these raw data is required to be preprocessed before the actual conversion operation. Because they strongly correlate to spatial location, it is well suited to use GIS ideal and function to achieve streamline processes of source data, thereby enhancing all aspects of coherence. Here, data preprocessing includes format conversion, projection transformation, treatment of semantic consistency, property assignment, etc. All of these work, either can be achieved directly by using GIS, or the implementation can be accelerated by making use of GIS functionality indirectly.

Data from different departments often use different organization rules, and use different formats to store. In order to use these data, we must first solve the problem that their formats are inconsistent. Commercial GIS software provides import and export functions for numerous spatial data formats, especially majority of domestic GIS software support common formats such as shapefile, TIF, which can greatly enhance the ability to spatial data exchange. Format conversion enables multi-source data integration and related processing become possible.

All digital spatial information is always constructed based on a certain coordinate systems. As previously described, we used to carry out spatial information collected based on different coordinate systems at different times. Meanwhile, different departments are unconsciously using different coordinate systems to produce their own business data. Now projection transformation theory and technology become more mature, with which GIS can provide fast coordinate systems transformation for vertical datum transformation studies.

Due to differences in professional background and the practical needs of industry applications, the definition of the same geographic entity comes from different departments is often different. Before utilizing these two types of data to carry out vertical datum conversion research, the inconsistency must be eliminated. Typically, topographic maps mainly organizes data according to geosciences classification, but marine maps still need to consider the actual needs of navigation (Huang zhonggang, 2009). GIS experts have done a lot of research in this area, and their findings can be used directly or indirectly in this area .

Tidal stations play an important role in land elevation datum and sea depth datum transformation, so we usually use them to observe long-term changes in water level. And the depth datum can be determined by level data analysis. As GIS has series of strong spatial query functions, they can be used to quickly achieve geographic relationship with varies of businesses. After the data associated with geography, they can really play their roles in the follow-up study. Meanwhile, the achievement of spatio-temporal data management and query research can also help to achieve efficient utilizing for multi-temporal data from tidal stations.

Data quality is an important factor affecting the success of the study. Quality of spatial data includes data integrity, data logical consistency, positional accuracy, attribute accuracy, time accuracy, etc. Among these problems, some are difficult to be found, only can be achieved by a lot of comparison and verification, such as integrity and logical consistency. Some are also affected by other factors, for example, location accuracy associated with time accuracy. Early data quality checking can help to discover and solve potential problems, ensure the quality of raw data, and avoid repeated work later. 


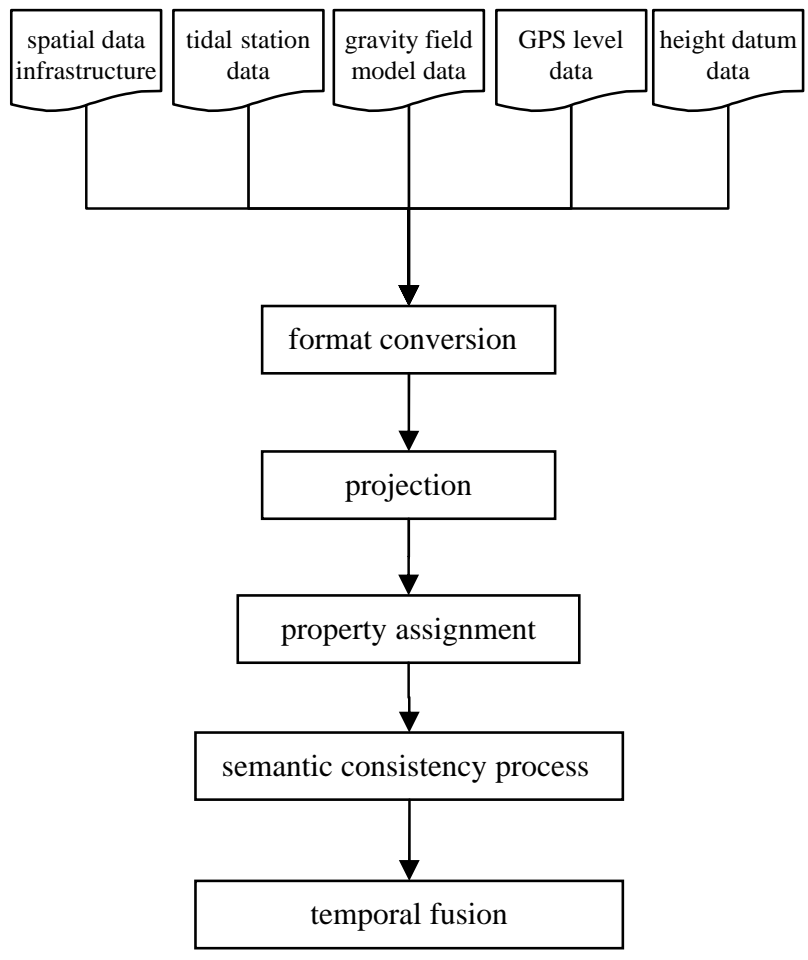

Figure 1 Raw data preprocessing

\subsection{Data management}

The data for vertical datum study has obvious features such as multi-source, multi-temporal state, multi-semantic, and multiscale. Meanwhile, during the course of the study, trial and analysis need to be repeated until the research goals are achieved. So as involvement of various data, efficient management and rapid application is important to carry out the research of vertical datum transformation successfully.

Firstly, using GIS, we can achieve the integrated management of raw data and resultant data. GIS is experienced in data integrity for storage management in the object-oriented approach, and there are many proven solutions. On the one hand, this integrated management can help us completely record the entire research process. On the other hand, it also can help us recover the whole course of the research at any time, which is very important to summarize and analysis the findings. For example, level data from tidal station is usually used after interpolation, and many interpolation algorithms are available. Different algorithms are suitable for different geographical environments, and produce different results. Meanwhile, the same algorithm with different input parameters may lead to different result. Therefore, reliable storage management for raw data is the basis for smoothly and deepening implementation of scientific work. Because most data in vertical datum conversion research is directly and indirectly location-related, so it is necessary to use GIS to manage the research data.

Secondly, on the one hand vertical datum conversion research need to use long-term observations data from tidal stations, how to effectively manage these long-state data is the problem we have to solve. On the other hand, the researchers sometimes do not have enough data coverage for the target area over the same period. How to use temporal GIS technology to reconstruct full coverage data records over the same period is crucial to carry out rigorous scientific research. For the former, there are more in-depth studies of temporal GIS in intelligent transportation, land management, and already have series of mature application cases. On this basis, according to features of level monitoring data from tidal stations, object-oriented management of monitoring data can be achieved by some necessary extensions. For the latter case, GIS researchers are studying general theory and techniques for long-state spatial data fusion, and have made some breakthroughs. Algorithms such as thiessen polygons, inverse distance weighted, gradient inverse distance, splines, trend surface area, ordinary kriging, are extended to conduct spatio-temporal interpolation. Achievement of these studies can be used for tense unity of non-full-coverage and long-time sequence monitoring data.

Thirdly, the data for vertical datum conversion study is usually collected by different departments with various organizations. Therefore, multi-format accessing support will help us to speed up the research process. As GIS is the most widely used information integration platform, it has a number of data interfaces to access various of formats, including SHAPEFILE, TIF, IMG, GEODATABASE, etc. These interfaces can be directly used, and geodesy community can focus on their research without worrying the data format. By means of GIS, we can quickly take advantage of various types of geospatial data, no matter origin data nor result data, can be conveniently shared with other departments.

Fourthly, efficient spatial retrieval can help build conversion model smoothly. Theoretically, the vertical datum is seamless continuous, and reference value of some points in blank area are more greatly impacted by certain observations known around it. Adjacent point query around a known point is a process of space optimal solving. GIS has strong spatial query and topological analysis capabilities, with which we can build a vertical datum model which can better meet the specific topography features, and the research results will be more scientific, and more in line with the laws of nature.

\section{INTERACTIVE MODELLING}

Vertical datum modelling involves many data sources, and is a continuous process of approximation. Specifically, on the one hand the number of measurements is large and widely distributed. On the other hand, measurements have complex effects on the unknown target point. For example, it is very useful to conduct the cause analysis and model optimization in vertical datum modelling process with the help of interactive modelling with graphical interface that can help us quickly find the optimal solution.

\subsection{Model visualization}

For complex modelling exercise, there are many types of experiment data. How to understand these data efficiently, as well as analyze the potential relationship between them is important to researchers. Model visualization can help researchers to quickly grasp the overall situation of modelling. It includes the original data visualization and result data visualization.

Although the data that we contacted is some description of natural and human environment, usually it is information stored in binary mode. It cannot be directly perceived, and usually is shown by some visual way. To convert this binary digital signal to intuitive picture, we need to go through three-step processing including data retrieval and pre-processing, symbolic, cartographic output. Each step of above three includes a variety of other technologies. For example, usually database technology is used for achieving attribute query, and topology operation is used for spatial query. Meanwhile, efficiency of data retrieval is an important problem, and it can be achieved by building properties and spatial indexes which is what GIS is 
good at. If high-quality visualization is required, each step of the processing will be different. GIS originated in cartography, and it is the development of cartography in the information age. For a long time, cartography committed clearly and intuitively painting for natural and social phenomena of the Earth's surface within a certain region according to some mathematical law, provisions schemata of symbols and colors. Over nearly 20 years, GIS experts have conducted a lot of studies in the map information, maps information transferring, maps feelings, graphic symbols and map models. With these studies, fruitful results have been achieved.

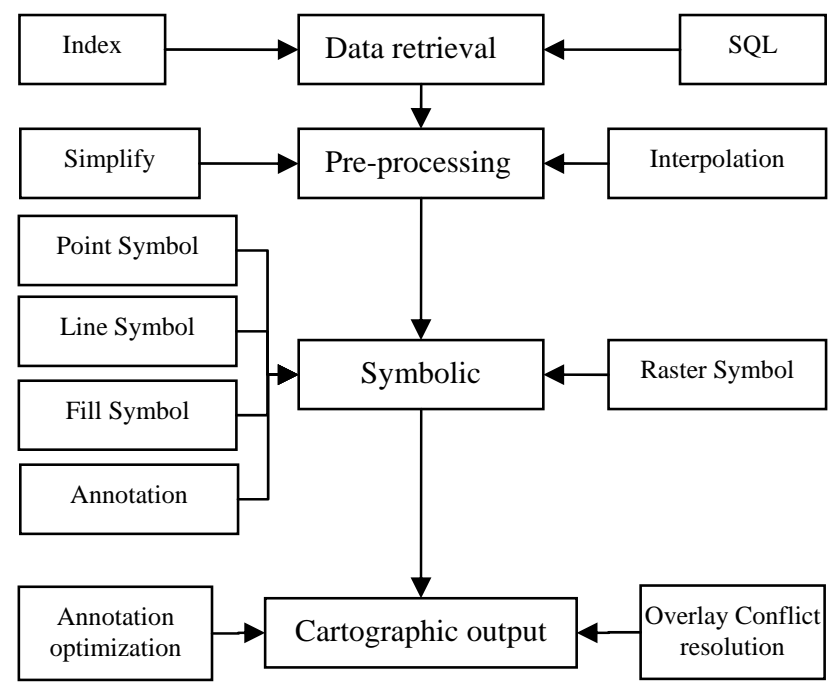

Figure 2 Data Visualization workflow

According to the natural characteristics of target elements, the original data are rendered by deploying conventional cartographic symbolic standards, and the example of visualization results is shown as below.

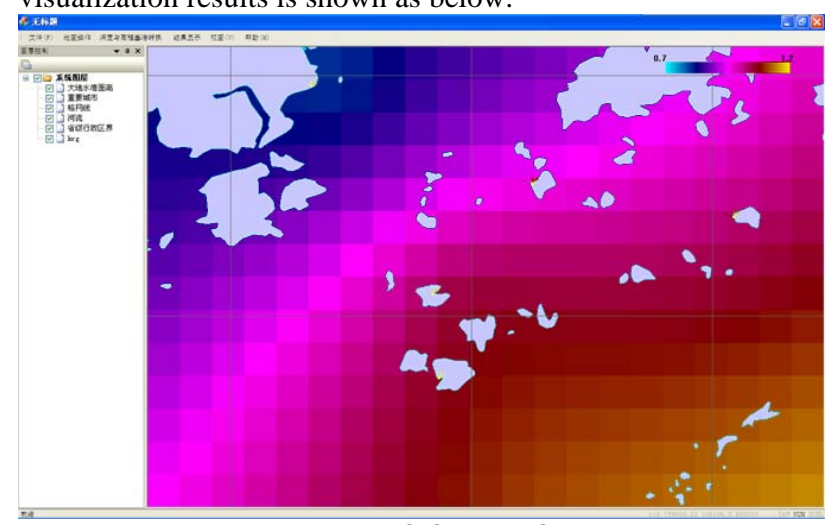

\subsection{Geographic association analysis}

Generally, vertical datum modelling is carried out in a large area, with larger amount of data and complex terrain environments.

\subsubsection{Assisted modelling}

Vertical Datum modelling research will introduce a variety of interpolation algorithms. At the same time, small changes of their input parameters may bring significant changes in the computed results. There is an urgent need of a mechanism to resolve these problems.

Overall, vertical datum modelling is relatively complex, as which use various algorithms to analyze various types of georeferenced data. Building clear and complete process workflow is very meaningful. By using plentiful data access interfaces of GIS, tidal station data, gravity field model data, GPS level data, and vertical datum data are processed completely. Then they are reconstructed with object-oriented approach which is commonly accepted by GIS community, and widely used in the field of GIS. Currently, all of the mature domestic GIS software is developed in the object-oriented manner. The objects reconstructed from origin data are then passed to various types of model computation. After specific calculation and analysis, the final results are outputted in standard GIS formats with which visual analysis can be well developed subsequently. Realizing seamless convergence of all aspects is the workflow we usually refer to. In this case, researchers can focus on the input of source data, while the middle process can be completed by the computer automatically. This will greatly optimize our study, and it is also the purpose of this study lies on.

With the aid of GIS, vertical datum modelling workflows can be constructed, and research efficiency can be improved greatly. But for specific treatment of certain aspects, such as interpolation, if the application is a combination of a variety of methods, the first problem we must face is that how they collaborate, and how to assign their respective roles. Generally this is often not an easy decision we can make, which often need continue to improve until the research goals are reached after numerous tests are going through. This involves a number of issues, such as the effect weight and impact scope for each method. Single weight handle can be simply implemented by changing its value directly. But if impact scope is considered, the worse is the unknown point not only affected by the closest known point, but also changing with different distance. It is a chain reaction, workload of artificial solver is huge. However, it is the strength of GIS, which can be very easy to implement by using topological analysis.

4.2.2 Verification of the results

Results of vertical datum modelling are abstract. Typically, intuitive visualization can help researchers quickly understand the overall of research results. But verification of the results need further processing, which includes two forms, one is comparing the accuracy between calculated values and measured values, the second is analysing its scientific according to surrounding environmental factors. Usually the former includes automatic and manual interaction. Their processes are similar, firstly get the spatial location of the target point, and then use spatial query to retrieve the calculated values of target point, finally compare calculated values with measured values, and derive the accuracy. Efficient visualization and interactive spatial query are basic functions that all GIS software must possess, which can be applied to verification of these results.

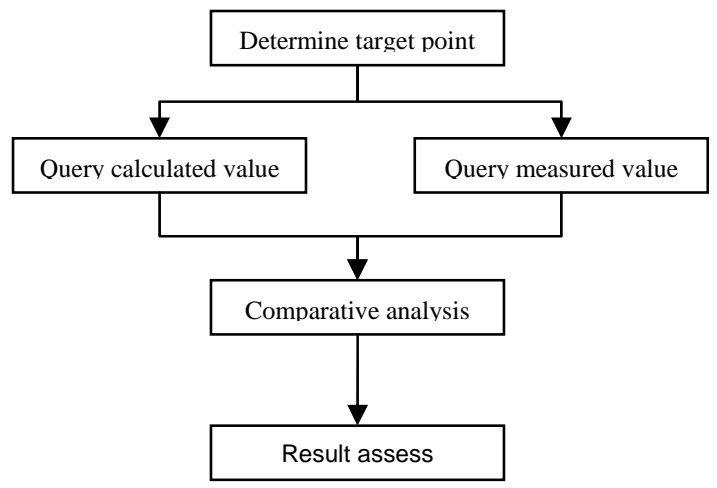

Figure 4 Accuracy assessment

Compared with accuracy assessment for known point, scientific result analysis in unknown region is more difficult, often 
requires large amounts of reference data, and use expert knowledge to conduct in-depth analysis. There are generally two ways which can help us to carry out this task, one is interpolating a region-wide and seamless vertical datum model based on measured data. The other is analyzing the topography and other environmental characteristic surrounding the measured location, then tap the potential links between topography on specific geographic location and vertical datum model. Based on this, we can analyze the computed results. Throughout the whole process, basic functions of GIS, such as spatial data reading, spatial querying, and topological computing are frequently used, and improve the efficiency of the research work. Meanwhile, an interactive information query environment with map visualization can greatly improve the research environment.

\section{CONCLUDING REMARKS}

With the in-depth application of geographic information in our working and living, the contradiction between explosive growth in the absolute amount of space information and relative lack of information which can be used directly, has become increasingly prominent. There are urgent needs for multi-source data fusion, and the most important one is vertical datum unification. But the transformation studies need to analyze large amounts, multi-type, multi-temporal state, and multi-scale spatial data with a variety of methods. How to achieve integrated management of these data, as well as the associated application and efficient operation of various methods, is what geodetic scientists must consider currently. Based on rich and powerful spatial data management, spatio-temporal query, topological analysis, cartographic visualization capabilities of GIS, system management for the whole process of vertical datum conversion modelling can be implemented easily.

\section{Acknowledgements}

The authors would like to thank the financial support from 863 High-Tech Project (no. 2013AA122502-4).

\section{References}

Guo chunxi, Nie jianliang, Wang bin, Jiang guangwei. The Fitting of GPS-Level and Gravity Quaigeoid Based on Adaptive Collocation[J]. Geomatics and Information Science of Wuhan University. 2013, 38(1):44 47.

Yang qingzhen, Guo chunxi. The Comparation of Multiple Fitting Methods Used in Quasi-geoid Refinement[J]. Standardization of Surveying and Mapping, 2012 (1): 30-32.

Huang Zhonggang, Wei Jintao, Ji Hongyu, Chen Jingjing, Ning Fanghui, Research on Data Structure Reference Topographic Maps to Charts[J]. HYDROGRAPHIC SURVEYING AND CHARTING, 2009, 29(3):58 60,64.

LI Jiancheng. The Recent Chinese Terrestrial Digital Height Datum Model: Gravimetric Quasi-Geoid CNGG2011[J]. Acta Geodaetica et Cartographica Sinica, 2012, 41(5):651 660,669.

Wang jiayao, Wei haiping, Cheng yi, Xiong ziming. The Research and Development of Spatio-temporal GIS[J]. HYDROGRAPHIC SURVERING AND CHARTING. 2004, 24(5):1 4.
Yang yuanxi, Liu nian, A Kind of Approximation Method on Gravity Anomaly[J]. Acta Geodaetica et Cartographica Sinica, 2001, 30(3):192 196.

Zhang xiaohong, Cheng shilai, Xu xiaodong. Research of GPS Elevation Fitting Models based on Kriging Method. JOURNAL OF GEODESY AND GEODYNAMICS. 2007, 27(2):47 51 